\title{
Análise Comparativa de Classificadores em Interfaces Cérebro- Computador Baseadas em SSVEP
}

\begin{abstract}
João Paulo S. P. Pereira (IC), Thiago B. S. Costa (PG), Sarah N. Carvalho (PQ), Romis Attux (PQ) Resumo

Interfaces cérebro-computador (BCls, do inglês brain-computer interfaces) são sistemas capazes de permitir a um usuário, por meio da análise de seus padrões cerebrais, o envio de comandos a uma máquina. $O$ desenvolvimento dessa tecnologia promete mudanças importantes no potencial de acessibilidade trazido pela moderna tecnologia informática. Neste projeto, buscamos analisar o desempenho de diferentes paradigmas de classificação em sistemas baseados em SSVEP (do inglês, Steady-State Visual Evoked Potentials), incluindo máquinas de aprendizado extremo (ELMs).
\end{abstract}

Palavras Chave: Interfaces Cérebro-Computador, EEG, Inteligência Computacional

\section{Introdução}

BCls são dispositivos capazes de converter sinais cerebrais em comandos associados à intenção de um usuário humano. A tarefa fundamental de uma $\mathrm{BCl}$ é identificar a intenção do usuário a partir de um conjunto limitado de sinais cerebrais, permitindo a realização de controle via dispositivos eletrônicos [1].

SSVEPs são respostas naturais a estímulos visuais com oscilação periódica estacionária. As frequências de estímulo geralmente são escolhidas na faixa de 5 a $30 \mathrm{~Hz}$, devido a suas propriedades favoráveis à detecção [2]

Para estudar os fundamentos de uma $\mathrm{BCl}$ baseada em SSVEP, implementou-se uma plataforma para operação offline contendo todas as etapas de uma interface desse tipo: préprocessamento, extração de características e classificação. Esta última etapa constitui o foco do projeto.

\section{Resultados e Discussão}

A implementação da plataforma (offline) baseada no paradigma de SSVEP teve como objetivo o processamento do sinal cerebral de entrada de forma que fosse possível determinar para qual frequência de estimulação o usuário estava olhando em um dado momento. O sinal foi obtido através de EEG, amostrado a $256 \mathrm{~Hz}$, registrado através de 16 eletrodos localizados no couro cabeludo. O experimento foi aprovado pelo Comitê de Ética da UNICAMP.

Como técnica de pré-processamento, adoutou-se uma filtragem espacial baseada em CAR (do inglês, Common Average Reference). Através desse método, o valor médio dos 16 eletrodos que compunham o sinal era subtraído de cada canal para cada uma das amostras.

Para a extração de características, utilizou-se o método de periodograma de Welch, através do qual se obteve uma estimativa da densidade espectral do sinal.
Foram testados 2 métodos distintos de classificação linear: mínimos quadrados e discriminante de Fisher. $\mathrm{O}$ primeiro busca encontrar o melhor ajuste para um conjunto de dados, tentando minimizar a soma dos quadrados das diferenças entre o valor estimado e os dados observados. Já o método de discriminante de Fisher, baseia-se nos princípios de maximizar a distância entre as classes a serem classificadas e, ao mesmo tempo, minimizar a distância entre os dados de uma mesma classe.

Os métodos de classificação não-lineares de ELM com e sem regularização foram também implementados e testados. $\mathrm{Na}$ tabela 1 encontram-se as taxas de acerto obtidas para cada um dos classificadores testados. As frequências analisadas foram as de 20 e $30 \mathrm{~Hz}$.

Tabela 1. Taxas de acertos obtidas

\begin{tabular}{|c|c|c|c|}
\hline $\begin{array}{c}\text { Mínímos } \\
\text { Quadrados }\end{array}$ & $\begin{array}{l}\text { Discriminante } \\
\text { de Fisher }\end{array}$ & ELM & $\begin{array}{c}\text { ELM com } \\
\text { regularização }\end{array}$ \\
\hline $83.35 \%$ & $83.35 \%$ & $79.61 \%$ & $87.51 \%$ \\
\hline
\end{tabular}

\section{Conclusões}

No projeto realizado, procurou-se uma compreensão dos aspectos básicos, sob os pontos de vista teórico e prático, envolvidos na construção de $\mathrm{BCls}$ baseadas no paradigma de potenciais evocados visualmente. Dos métodos de classificação analisados, aquele que forneceu a melhor taxa de acertos foi o de ELM com regularização

\section{Agradecimentos}

Somos gratos ao PIBIC e ao CNPq pelo financiamento deste projeto e à equipe do DSPCOM pelas inúmeras contribuições técnicas.

[1] U. Hoffmann, J. M. Vesi, T. Ebrahimi e K Diserens, “An efficient P300-based brain-computer interface for disabled subjects", Journal of Neuroscience methods, v. 167, n. 1, pp. 115-125, 2008.

[2] G. Garcia Molina, "Detection of high-frequency steady state visual evoked potentials using phase rectified reconstruction."

16th European Signal Processing Conference, EUSIPCO, 2008.. 